\title{
Analysis and Scheduling of Maintenance Operations for a Chain of Gas Stations
}

\author{
Mehmet Savsar \\ Kuwait University, College of Engineering and Petroleum, P.O. Box 5969, 13060 Safat, Kuwait \\ Correspondence should be addressed to Mehmet Savsar; msavsar@gmail.com
}

Received 6 November 2012; Revised 2 February 2013; Accepted 3 February 2013

Academic Editor: Anis Chelbi

Copyright (C) 2013 Mehmet Savsar. This is an open access article distributed under the Creative Commons Attribution License, which permits unrestricted use, distribution, and reproduction in any medium, provided the original work is properly cited.

\begin{abstract}
Maintenance is one of the central issues in operational activities, which involve any type of equipment. In this paper we have considered analysis, modeling, and scheduling of preventive maintenance operations for fuel dispensers in a chain of gas stations. A gas station company with more than 570 dispensers in more than 40 stations is considered and the maintenance problem is studied in detail. Operations research tools, including maintenance models and linear programming, were used to establish optimum schedules for preventive maintenance operations. Detailed cost analyses were carried out to determine feasibility of the proposed preventive maintenance schedules. Models and procedures presented in this paper could guide operation engineers and maintenance managers in solving similar problems for operational improvements.
\end{abstract}

\section{Introduction}

Complex equipment and devices used in any system constitute majority of the capital invested in industry. Equipment is subject to deterioration with usage and time and deterioration is often reflected in higher operation costs and lower service quality. In order to keep operational costs down while maintaining good service quality, preventive maintenance (PM) is often performed on a scheduled basis. The cost of maintenance-related activities in industrial facilities has been estimated by Mobley [1] as $15-40 \%$ of total operation costs and the trend toward increased automation has forced managers to pay even more attention to maintain complex equipment and keep them in available state. If the equipment is maintained only when it fails, it is called corrective maintenance $(\mathrm{CM})$, while preplanned maintenance is called preventive maintenance (PM). Traditionally it is known that the probability of failure would increase as equipment is aged, and that it would sharply decrease after a planned preventive maintenance (PM) is implemented. However, as indicated by Savsar [2], the amount of reduction in failure rate due to introduction of a preventive maintenance has not been fully studied. In particular, it would be desirable to know the performance of a system before and after the introduction of PM. It is also desired to know the type and the rate at which a preventive maintenance should be scheduled or the maintenance policy to be implemented.

A gas station includes several facilities and equipment that need to be maintained. In particular, dispensers (gasoline pumps), storage tanks, car wash equipment, and other ancillary equipment need to be kept in operational condition for effective performance and profitable service. The first gasoline pumps were developed in 1885 in Indiana by $\mathrm{S}$. F. Bowser to be used for kerosene lamps and stoves. Later, when the automobiles were invented, these pumps were improved and used by adding a hose and several other safety measures. A modern fuel dispenser is divided into two main parts, including an electronic head and a mechanical section. Electronic head contains an embedded computer, which controls the action of the pump, drives the pump's displays, and communicates with an indoor sales system. The mechanical section, which is in a self-contained unit, has an electric motor, pumping unit, meters, pulsers, and valves to physically pump and control the fuel flow. There are many different variations of fuel dispensers in use today. The term "gas pump" is usually used as an informal way to refer to a fuel dispenser.

In order to maintain functionality of a gas station, all equipment has to be maintained by preventive maintenance at scheduled times or repairs when failures occur. Every fuel 
dispenser is a combination of various small components and it is essential to ensure the appropriate performance of each component for profitable and high quality service in a gas station. Typical maintenance activities in a gas station include

(i) mechanical pumps, meters, and lubrication equipment repair,

(ii) electronic dispenser and electronic control console repair,

(iii) automated system repair including automatic tank gauging, release detection systems, and Point-of-Sale systems,

(iv) (POS), price scanners, card readers, and communication links,

(v) tank system repair including: tanks, pumps, leak detectors, piping, hoses, and nozzles,

(vi) car wash system and ancillary equipment maintenance and repairs.

Preventive maintenance (PM) programs are implemented to reduce annual repair costs and costs associated with equipment downtime. While preventive maintenance and repair instructions of major equipments at gas stations are specified by the manufacturers, it is difficult to find specific studies related to maintenance analysis of such systems. However, extensive studies have been carried out in the areas of reliability and maintenance management in general. The existing body of theory on general system reliability and maintenance is scattered over a large number of scholarly journals belonging to a diverse variety of disciplines. In particular, mathematical sophistication of preventive maintenance models has increased in parallel to the growth in the complexity of modern manufacturing systems. Research work has been published in the areas of maintenance modeling, optimization, and management. Cho and Parlar [3] presented surveys of maintenance models for multiunit systems. Dekker [4] also presented an excellent review of maintenance optimization models. Sheu and Krajewski [5] presented a decision model based on simulation and economic analysis for corrective maintenance policy evaluation. Simeu-Abazi et al. [6] evaluated dependability of manufacturing systems. Waeyenbergh et al. [7] presented a case study for maintenance concept development. Also, Waeyenbergh and Pintelon [8] discussed procedures, knowledge-based concepts, and frameworks in maintenance policy development and implementation in industry. Komonen [9] presented a cost model of industrial maintenance for profitability analysis. Abdulmalek et al. [10] presented a simulation model and procedures for analyzing tool change policies in a manufacturing system. Chan et al. [11] also presented a maintenance implementation for total productive maintenance in the context of a case study in electronics industry. Kyriakidis and Dimitrakos [12] presented a maintenance model for a production system with intermediate buffers. Gómez de León Hijes and Cartagena [13] presented a maintenance strategy based on equipment classification using a multicriterion objective. Carnero [14] presented a procedure for setting up a predictive maintenance program using detailed system evaluation. Savsar [15] and
Savsar and Aldaihani [16] presented reliability models for flexible manufacturing cells (FMC) and obtained FMC availability assuming only corrective maintenance is performed. Savsar $[17,18]$ presented models for analysis of the effects of maintenance policies on FMC performance measures. Savsar [19] developed a discrete mathematical model to describe a serial production flow line with buffers and machine failures and incorporated it into a simulation model to study the effects of equipment failures and corrective maintenance operations on production line performance under different operational conditions.

While most of the studies related to reliability and maintenance analysis are theoretical, limited numbers of practical applications are published in the literature. In this study, maintenance modeling and analysis is carried out in the context of a chain of gas stations system. In particular, models are presented for determination of various maintenancerelated activities. It is well known that equipment failures occur due to wearouts and random causes. Therefore, in the most general way, maintenance operations are classified as corrective maintenance $(\mathrm{CM})$ and preventive maintenance (PM). There are some other variations of these maintenances. However, CM and PM are the most general procedures in industry. Causes of random failures, which result in CM, are not known and cannot be predicted. These types of failures occur even in new systems. However, wearout failures occur by the usage of the equipment and as the time passes. These failures can be predicted and mean time to failure can be estimated. PM operations are carried out before the expected failures. For example, time to failure of a gear or a belt due to usage can be estimated and the time to change the component can be specified. While random failures cannot be eliminated totally, wearout failures can be eliminated by PM operations and thus a reduction in CM can be achieved. The exact effects of PM operations in reducing CM frequency are modeled and applied to various components in the selected system. System down time and productivity are estimated before and after the introduction of PM. Finally, cost analysis of the system is carried out with respect to the effectiveness of PM operations. Models and analysis developed and presented in this paper will be useful for maintenance engineers and operation managers in practical applications.

\section{Analysis of Preventive Maintenance Operations for Fuel Dispensers}

The gas station company considered in this study had a chain of 41 gas stations and a total of 577 dispensers. In order to perform the maintenance analysis, data related to dispenser failures at each gas station were collected and gas stations were grouped into three categories as high-failure stations, medium-failure stations, and low-failure stations. Grouping was necessary since it was not practical to analyze each of 41 stations separately. Each group was analyzed based on average failure and maintenance rates in order to have applicable results.

It is well known that performing preventive maintenance at scheduled points in time before an asset loses optimum 
performance can help in providing acceptable levels of operability in efficient and cost-effective manners. As the preventive maintenance is increased, the need for corrective maintenance is reduced and subsequently, the down time of the equipments will be reduced too. A study of rescheduling the preventive maintenance was performed by analyzing the effect of increasing the preventive maintenance on the mean time between failures.

The study was applied on the dispenser area for the three categories of high-, medium-, and low-failure stations. The compound mean time between maintenance activities, $\mathrm{MTBM}_{\mathrm{mt}}$, must be obtained by combining the rates for corrective maintenance $(\mathrm{CM})$ and preventive maintenance (PM) activities. Assuming that the CM and PM activities are independent, the compound maintenance rate for $\mathrm{CM}$ and PM activities is obtained by the adding the related rates. The following notations are used for the calculations:

\section{$\lambda$ : corrective maintenance rate, \\ $\pi$ : preventive maintenance rate,}

$\operatorname{MTBM}_{c t}=1 / \lambda$ : mean time between corrective maintenance (this is also referred to as MTBF),

$\operatorname{MTBM}_{\mathrm{pt}}=1 / \pi$ : Mean time between preventive maintenance.

Combined maintenance rate is obtained from $\lambda+\pi$ and compound mean time between all maintenances is calculated from $1 /(\lambda+\pi)$, which can also be expressed as

$$
\mathrm{MTBM}_{\mathrm{mt}}=\frac{1}{1 / \mathrm{MTBM}_{\mathrm{ct}}+1 / \mathrm{MTBM}_{\mathrm{pt}}}=\frac{1}{\lambda+\pi} .
$$

This equation shows the relation between mean time between maintenance activities and the maintenance rates. It does not depend on the distribution of failures. Time between failures can follow gamma distribution and its special form, which is exponential distribution.

Mean rate of compound maintenance is defined as $\psi=$ $1 / \mathrm{MTBM}_{\mathrm{mt}}$.

Failure rate can be expressed as a function of combined maintenance rate and the preventive maintenance rate by manipulating (1) as follows:

$$
\lambda=\frac{1}{\mathrm{MTBM}_{\mathrm{mt}}}-\pi
$$

where $\pi=1 / \mathrm{MTBM}_{\mathrm{pt}}$. Since increasing preventive maintenance decreases the need for corrective maintenance, it is possible to alter the PM rate $\pi$ in order $o$ recalculate the estimated corrective maintenance. In this case, combined maintenance rate is kept constant and CM rate is estimated based on a given PM rate. Maintenance analysis is carried out for dispensers in each group of stations. Value of $\lambda$ and $\pi$ can be easily estimated in a real system. Time between failures of a component can be recorded over time and the mean time between failures can be estimated from the data. Failure rate can be estimated for the component or the system under consideration by $\lambda=1 / \mathrm{MTBF}$. Maintenance rate, $\pi$ can also be easily estimated based on mean time between preventive maintenances of the component or the system considered based on the expected MTBMs specified by the manufacturer.
TABLE 1: Increasing $\mathrm{MTBM}_{\mathrm{ct}}$ as a result of decreasing $\mathrm{MTBM}_{\mathrm{pt}}$ for high failure stations.

\begin{tabular}{lc}
\hline MTBM $_{\mathrm{pt}}$ & MTBM $_{\mathrm{ct}}$ (hours) \\
\hline 4 months & 459 \\
3 months & 485 \\
2 months & 546 \\
1 month & 877 \\
\hline
\end{tabular}

2.1. High Failure Stations. High failure stations category consists of 7 stations with a total of 133 dispensers. The average failure rate was found to be $\lambda=0.002179$ failure/hour/ dispenser which equals 2539 failures per year. Currently, $\mathrm{MTBM}_{\mathrm{pt}}$ equals 4 months (30 days per month and 24 hours per day) and the preventive maintenance rate was calculated as follows:

$$
\pi=\frac{1}{4 * 30 * 24}=0.000347 .
$$

By using the failure rate and the preventive maintenance rate, combined maintenance rate, $\mathrm{MTBM}_{\mathrm{m}}$, was calculated using (1) and found to be 395.88 hours. By substituting the $\mathrm{MTBM}_{\mathrm{mt}}$ into (2) and changing the PM rate, several possible failure rates were estimated using the equation below:

$$
\lambda=\frac{1}{395.88}-\pi \text {. }
$$

Table 1 summarizes the $\mathrm{MTBM}_{\mathrm{ct}}$ for different preventive maintenance schedules. From the results in the table, it can be shown that for the high failure stations, $\mathrm{MTBF}_{\mathrm{ct}}$ increases slightly when performing the preventive maintenance once every two months, but it increases highly when performing preventive maintenance once every month. Effectively, failure rate $\lambda=1 / \mathrm{MTBM}_{\mathrm{ct}}$ decreases significantly as $\mathrm{PM}$ is performed more frequently, such as every month.

2.2. Medium Failure Stations. Medium failure stations category consists of 17 gas stations with a total of 248 dispensers. The average failure rate was found to be $\lambda=0.001935$ failure/hour/dispenser which equals 4204 failures per year. Currently, $\mathrm{MTBM}_{\mathrm{pt}}$ equals 4 months and the preventive maintenance rate was calculated as follows:

$$
\pi=\frac{1}{4 * 30 * 24}=0.000347 .
$$

By utilizing the failure rate $\lambda$ and the preventive maintenance rate $\pi$, combined maintenance rate, $\mathrm{MTBM}_{\mathrm{mt}}$, was calculated using (1) and found to be 438.212 hours. Finally, by utilizing this value and different values for PM schedules, a set of possible corrective maintenance rates were estimated using the equation below and tabulated in Table 2. From Table 2, it can be shown that for the medium failure stations, the $\mathrm{MTBM}_{\mathrm{ct}}$ increases slightly when performing the preventive maintenance once every two months, but it again increases sharply when performing the preventive maintenance once every month:

$$
\lambda=\frac{1}{438.212}-\pi .
$$


TABLE 2: Increasing $\mathrm{MTBM}_{\mathrm{ct}}$ as a result of decreasing $\mathrm{MTBM}_{\mathrm{pt}}$ for medium failure stations.

\begin{tabular}{lc}
\hline MTBM $_{\mathrm{pt}}$ & MTBM $_{\mathrm{ct}}$ (hours) \\
\hline 4 months & 517 \\
3 months & 549 \\
2 months & 629 \\
1 month & 1121 \\
\hline
\end{tabular}

2.3. Low Failure Stations. Low failure category of stations consists of 17 gas stations with a total of 196 dispensers. The average failure rate was found to be 0.00122 failure/hour/dispenser which equals 2095 failures per year. Currently, MTBM $_{\mathrm{pt}}$ equals 4 months and the preventive maintenance rate was determined as follows:

$$
\pi=\frac{1}{4 * 30 * 24}=0.000347 \text {. }
$$

By utilizing failure rate and preventive maintenance rate, combined maintenance rate, $\mathrm{MTBM}_{\mathrm{mt}}$, was calculated using (1) again and found to be 638.162 hours. Finally, failure rates were recalculated based on various values of preventive maintenances using the equation below:

$$
\lambda=\frac{1}{638.162}-\pi,
$$

Table 3 summarizes the $\mathrm{MTBF}_{\text {ct }}$ for different PMs. From Table 3, it can be seen that for the low failure stations, $\mathrm{MTBF}_{\mathrm{ct}}$ increases slightly when performing the PM up to once every two months, but it increases again sharply when performing $\mathrm{PM}$ once every month. These results will be used in the next section to optimize PM schedules for the dispensers in three categories of stations.

\section{Preventive Maintenance Scheduling for Fuel Dispensers Using LP Model}

From the previous analysis, we found that the $\mathrm{MTBF}_{\mathrm{ct}}$ for $\mathrm{CM}$ increases as the mean time between PM is decreased. But, we have some constraints that prevent us from performing the $\mathrm{PM}$ once every month for the 41 gas stations. In particular, numbers of technicians are limited and the PM cannot be performed on more than one station per day; also it is not preferred to perform the PM monthly for the dispenser area. As a result, by comparing different situations for the three categories, it was found that the high failure stations have the lowest mean time between failures and the highest number of failures. Consequently, it was suggested to give them higher priority than the other stations.

A linear programming model was constructed to determine optimum PM schedule for the dispenser area. First, a pair wise comparison method was applied to assign weight to each failure category that will be used to formulate the objective function. Pairwise comparison is a kind of divide-and-conquer problem-solving method. It allows one to determine the relative ranking of a group of items. The first step is to identify the criteria. In our case we have three
TABLE 3: $\mathrm{MTBM}_{\mathrm{ct}}$ based on $\mathrm{MTBM}_{\mathrm{pt}}$ for low failure group of stations.

\begin{tabular}{lc}
\hline MTBM $_{\mathrm{pt}}$ & MTBM $_{\mathrm{ct}}$ (hours) \\
\hline 4 months & 820 \\
3 months & 909 \\
2 months & 1145 \\
1 month & 5650 \\
\hline
\end{tabular}

TABLE 4: Pair wise comparison matrix.

\begin{tabular}{lccc}
\hline & $\begin{array}{c}\text { High failure } \\
\text { stations }\end{array}$ & $\begin{array}{c}\text { Medium } \\
\text { failure stations }\end{array}$ & $\begin{array}{c}\text { Low failure } \\
\text { stations }\end{array}$ \\
\hline High failure stations & - & High & High \\
Medium failure stations & - & - & Medium \\
Low failure stations & - & - & - \\
\hline
\end{tabular}

criteria, such as high failure, medium failure, and low failure stations. The second step is to arrange the criteria in an $N \times N$ matrix and compare the item in the row with respect to each item in the rest of the row by putting the category that we consider the most important in each pair wise comparison. The matrix would be as shown in Table 4 .

The next step is to create the ranking of items by creating an ordered list of the items, ranked by the number of cells containing their names. This leads to

(i) high failure stations (2),

(ii) medium failure stations (1),

(iii) low failure stations (0).

In order to get the weights, we have assumed a linear proportion between all the weights and solved the equation below, which states that the total of all the weights must be $100 \%$. The weight assignments between classes or elements have been based on linear proportion in the literature unless there is evidence and data to assume some other relationship. In our case, we did not have any information or data that contradicts to the assumption of linear proportion between the weights. Therefore, we have used a linear proportion which is commonly used in the literature. The equation for the weights is as follows

$$
100=2 x+1 x+0 x .
$$

Therefore, $x=33.33$.

This leads to the following approximate weights:

(i) high failure stations: $60 \%$,

(ii) medium failure stations: $30 \%$,

(iii) low failure stations: $10 \%$.

Note that final percentages are approximated by rounding them down and the "10\%" for low failure stations resulted from summing the round-off values from other calculations. These final assignments after rounding down and up are arbitrary and do not necessarily carry a specific meaning or significance. The only importance is that we have to give 
TABLE 5: Various parameters and maintenance cost comparisons for 3 categories of dispensers.

\begin{tabular}{|c|c|c|c|c|c|c|c|}
\hline Category & $\begin{array}{c}\text { Number of } \\
\text { failures/year }\end{array}$ & $\begin{array}{c}\text { Number of } \\
\mathrm{PM} / \text { station/year }\end{array}$ & $\begin{array}{c}\text { Number of } \\
\text { stations }\end{array}$ & $\mathrm{CMC}$ & PMC & TMC & $\begin{array}{c}\text { Percent reduction } \\
\text { in cost }\end{array}$ \\
\hline High failure stations-under current PM & 3281 & 3 & 7 & 40161 & 1498 & 41695 & \\
\hline High failure stations - under proposed PM & 2747 & 6 & 7 & 33696 & 2992 & 36688 & $14 \%$ \\
\hline Medium failure stations-under current PM & 2101 & 3 & 15 & 25718 & 900 & 26688 & \\
\hline Medium failure stations-under proposed PM & 1395 & 4 & 15 & 17075 & 1199 & 18274 & $31 \%$ \\
\hline Low failure stations & \multicolumn{7}{|c|}{ Current and proposed PM schedules are the same and thus the costs are not affected } \\
\hline
\end{tabular}

some small weight to the low failure stations to represent them in the objective function, which will be shown below. After getting the weights, a linear programming model was formulated for maintenance scheduling. The following notations are used in the LP model:

$h$ : high failure stations; $h=1,2,3,4,5,6,7$,

$d$ : medium failure stations; $d=8,9,10,11,12,13,14$, $15,16,17,18,19,20,21,22,23,24$,

$l$ : low failure stations; $l=25,26,27,28,29,30,31,32$, $33,34,35,36,37,38,39,40,41$,

$m$ : months; $m=1,2,3,4,5,6,7,8,9,10,11,12$.

Objective Function. Consider:

$$
\begin{aligned}
\operatorname{Max} . \quad z= & 0.6 \sum_{h=1}^{7} \sum_{m=1}^{12} x_{h m}+0.3 \sum_{d=8}^{24} \sum_{m=1}^{12} x_{d m} \\
& +0.1 \sum_{l=25}^{41} \sum_{m=1}^{12} x_{l m} .
\end{aligned}
$$

Decision Variables. Consider:

$x_{h m}= \begin{cases}1 & \text { if station } h \text { is maintained in month } m \\ 0 & \text { otherwise, }\end{cases}$

$x_{d m}= \begin{cases}1 & \text { if station } d \text { is maintained in month } m \\ 0 & \text { otherwise }\end{cases}$

$x_{l m}= \begin{cases}1 & \text { if station } l \text { is maintained in month } m \\ 0 & \text { otherwise. }\end{cases}$

Constraints. Consider:

$$
\begin{aligned}
& \sum_{m=1}^{12} x_{h m} \geq 3 \quad \forall h, \\
& \sum_{m=1}^{12} x_{d m} \geq 3 \quad \forall d, \\
& \sum_{m=1}^{12} x_{l m} \geq 3 \quad \forall l, \\
& \sum_{m=1}^{12} x_{h m} \leq 6 \quad \forall h, \\
& \sum_{m=1}^{12} x_{d m} \leq 6 \quad \forall d,
\end{aligned}
$$

$$
\begin{gathered}
\sum_{m=1}^{12} \leq 6 \quad \forall l, \\
\sum_{h=1}^{7} x_{h m}+\sum_{d=8}^{24} x_{d m}+\sum_{l=25}^{41} x_{l m} \leq 13 \\
\forall m=3,4,6,8,9,11, \\
\sum_{h=1}^{7} x_{h m}+\sum_{d=8}^{24} x_{d m}+\sum_{l=25}^{41} x_{l m} \leq 14 \\
\forall m=1,5,7,12, \\
\sum_{h=1}^{7} x_{h m}+\sum_{d=8}^{24} x_{d m}+\sum_{l=25}^{41} x_{l m} \leq 12 \quad \forall m=2, \\
x_{h m}, x_{d m}, x_{l m}=\{0,1\},
\end{gathered}
$$

where the objective function maximizes the total number of preventive maintenances to be performed so that total corrective maintenances are minimized.

The first, second, and third constraints insure that the preventive maintenance is performed at least 3 times per year for the high failure stations, medium failure stations, and low failure stations, respectively (as the current situation).

The fourth, fifth, and sixth constraints insure that the preventive maintenance will not be performed every month, but at maximum of 6 times per year. This means that minimum time between PM will be 2 months.

The seventh, eight, and ninth constraints insure that the labor capacity in each month will not be exceeded, provided that it takes 2 days to perform the preventive maintenance in each station with the new schedule.

The model was solved using GAMS software and optimal preventive maintenance schedule was found to be as follows.

(i) For the high failure stations, 6 times per year.

(ii) For the medium failure stations, it was divided into three categories as

(a) 6 times per year for 3 stations,

(b) 4 times per year for 6 stations,

(c) 3 times per year for 8 stations.

(iii) For low failure stations, 3 times per year. 
As a result, 3 medium failure stations have the same schedule as the high failure stations and 8 medium failure stations have the same schedule as low failure stations. Therefore, we will assume that the 3 highest failure stations from the medium failure stations category will be added to the high failure stations, the 8 lowest failure stations from the medium failure stations category will be added to the low failure stations, and the remaining 6 stations will be kept as medium failure stations. In Section 5, a cost analysis will be performed to compare the optimum PM schedules with the current PM schedules.

\section{Maintenance Cost Analysis}

Preventive maintenance involves a basic tradeoff between the costs of conducting maintenance activities and the savings achieved by reducing the overall rate of occurrence of system failures. Although the gas company is paying a fixed amount of money per month regardless of the number of failures occurring per month, a study of the effects of changing the PM schedule on the total maintenance cost was performed to make sure that the new PM schedule will not cause them to pay more money. The total maintenance cost was found using:

$$
\mathrm{TMC}=\mathrm{CMC}+\mathrm{PMC}+\mathrm{DTC},
$$

where TMC is total maintenance cost per year

$\mathrm{CMC}$ is corrective maintenance cost per year calculated by

$$
\begin{aligned}
\mathrm{CMC}= & (\text { Number of Failures per Year }) \\
& *(\text { Corrective Maintenance Cost per Failure }),
\end{aligned}
$$

PMC is preventive maintenance cost per year calculated by

$$
\begin{aligned}
\text { PMC }= & (\text { Number of Preventive Maintenance per Year }) \\
& *(\text { Cost per Preventive Maintenance })
\end{aligned}
$$

DTC is down time cost per year calculated by:

$$
\begin{aligned}
\text { DTC }= & {\left[\left(\frac{\text { No. of failures }}{\text { year }}\right)\right.} \\
& *(\text { Average Repair Time + Average Waiting Time })] \\
& *[\text { Cost per Down Time hour }] .
\end{aligned}
$$

Total corrective maintenance cost for the dispenser area was provided by the gas company as $23,170 \mathrm{KD} /$ year. The total number of failures in the dispenser area for the 41 stations was 6869 failures/year. Thus, cost per failure, which resulted in corrective maintenance, was calculated by $23170 / 6869=$ $3.4 \mathrm{KD} /$ failure. This cost is the same for all categories of dispensers in all stations. Similarly, the PM cost for the dispenser area was provided by the gas station company as $1704 \mathrm{KD} /$ year. Currently PM is scheduled to be performed once every 4 months, or 3 times per year, in each of the 41 stations which resulted in a total of $3 \times 41=123$ PM per year. By dividing the PM cost by the total number of preventive maintenances, cost per preventive maintenance was found to be $1704 / 123=13.85 \mathrm{KD} / \mathrm{PM}$. This cost is also the same for all categories of dispensers in all stations. Downtime cost was not considered in the total maintenance cost based on the assumption that the sale will not be lost due to a dispenser failure as the customer will use another dispenser instead of leaving the system. Economic analyses are performed for each category of stations under the current (old) and proposed (new) PM policies.

4.1. High Failure Stations. As it was mentioned before, under the current PM schedule a PM is performed once every 4 months. As a result, high failure stations have 3281 failures/year, which results in $3281 \mathrm{CM}$ operations/year, and $3 \mathrm{PMs}$ per station per year. Equation (13) is utilized in cost calculations for dispensers in each category of stations. Note that 3 stations from the medium failure category are added to the high category due to same frequency of PM as obtained from LP model. Thus, using (13), maintenance costs for the current schedule for dispensers in high failure stations are calculated as $\mathrm{CMC}=$ 3281 failures/year $* 3.4 \mathrm{KD} /$ failure $=11156 \mathrm{KD} /$ year; $\mathrm{PMC}=$ $3 \mathrm{PM} /$ station/year $* 10$ stations $* 13.85 \mathrm{KD} / \mathrm{PM}=416 \mathrm{KD} /$ year; and $\mathrm{TMC}=11.572 \mathrm{KD} /$ year.

Under the proposed PM schedule, where PM is to be performed once every 2 months, the number of failures is expected to be 3047 failures/year and $6 \mathrm{PMs}$ are performed per station/year. Thus, the maintenance costs are calculated by (13) as $\mathrm{CMC}=$ 3047 failure $/$ year $* 3.4 \mathrm{KD} /$ failure $=10360 \mathrm{KD} /$ year; $\mathrm{PMC}=$ $6 \mathrm{PM} /$ station/year $* 10$ stations $* 13.85 \mathrm{KD} / \mathrm{PM}=831 \mathrm{KD} /$ year; and $\mathrm{TMC}=11.191 \mathrm{KD} /$ year. These maintenance cost for the current and proposed PM schedules are compared in Table 5, where system parameters are also listed. It can be seen that extra costs of PM is justified since the total costs are reduced from $11.572 \mathrm{KD}$ to $11.191 \mathrm{KD}$ for this category of stations.

4.2. Medium Failure Stations. For the medium failure stations, it was proposed to have a PM once every 3 months, that is, 4 times per year. Currently, PM is performed once every 4 months or 3 times per year. Thus, the maintenance costs for the current and proposed schedules are calculated as for high failure stations using (13) based on the parameters given in Table 5 . The final cost results are also listed in the table. Note that $3.4 \mathrm{KD} /$ failure and $13.85 \mathrm{KD} / \mathrm{PM}$ are common parameters for all cases. The cost results are compared in Table 5. PM costs are justified for this category also since total maintenance costs are reduced from $7.394 \mathrm{KD} /$ year to $5.076 \mathrm{KD} /$ year. 
4.3. Low Failure Stations. As it was mentioned earlier, PM was proposed to be performed once every 4 months, which is the same as the current schedule. Therefore, the costs would be the same for the current and the proposed schedules.

\section{Conclusions}

Maintenance is one of the major operational activities in industrial systems. In particular, implementation of a PM program requires detailed and careful analysis to justify the related costs since maintenance costs are a significant part of operational costs. Implementing a PM has to be justified by analyzing its effect on reducing CM costs. In this paper, a gas station company with a chain of 41 stations is considered and detailed procedures are presented for maintenance analysis. After analyzing the current system, a linear programming model is used to determine optimum PM activities to be performed for each category of gas stations. Furthermore, detailed cost analyses are performed for the dispensers to establish cost-saving PM schedules. LP model and the economic cost analysis procedures proved to be effective for the company considered. The models and procedures utilized in this paper could be used by operational engineers in maintenance analysis in other industrial settings in order to improve productivity and to reduce related operational costs.

\section{References}

[1] R. K. Mobley, An Introduction to Predictive Maintenance, Van Nostrand Reinhold, New York, NY, USA, 1990.

[2] M. Savsar, "Analysis and modeling of maintenance operations in the context of an oil filling plant," Journal of Manufacturing Technology Management, vol. 22, no. 5, pp. 679-697, 2011.

[3] D. I. Cho and M. Parlar, "A survey of maintenance models for multi-unit systems," European Journal of Operational Research, vol. 51, no. 1, pp. 1-23, 1991.

[4] R. Dekker, "Applications of maintenance optimization models: a review and analysis," Reliability Engineering and System Safety, vol. 51, no. 3, pp. 229-240, 1996.

[5] C. Sheu and L. J. Krajewski, "Decision model for corrective maintenance management," International Journal of Production Research, vol. 32, no. 6, pp. 1365-1382, 1994.

[6] Z. Simeu-Abazi, O. Daniel, and B. Descotes-Genon, "Analytical method to evaluate the dependability of manufacturing systems," Reliability Engineering and System Safety, vol. 55, no. 2, pp. 125-130, 1997.

[7] G. Waeyenbergh, L. Pintelon, and L. Gelders, "A stepping stone towards knowledge based maintenance," South African Journal of Industrial Engineering, vol. 12, no. 2, pp. 61-61, 2001.

[8] G. Waeyenbergh and L. Pintelon, "Maintenance concept development: a case study," International Journal of Production Economics, vol. 89, no. 3, pp. 395-405, 2004.

[9] K. Komonen, "A cost model of industrial maintenance for profitability analysis and benchmarking," International Journal of Production Economics, vol. 79, no. 1, pp. 15-31, 2002.

[10] F. Abdulmalek, M. Savsar, and M. Aldaihani, "Simulation of tool change policies in a flexible manufacturing cell," WSEAS Transactions on Systems, vol. 7, no. 3, pp. 2546-2552, 2004.

[11] F. T. S. Chan, H. C. W. Lau, R. W. L. Ip, H. K. Chan, and S. Kong, "Implementation of total productive maintenance: a case study,"
International Journal of Production Economics, vol. 95, no. 1, pp. 71-94, 2005.

[12] E. G. Kyriakidis and T. D. Dimitrakos, "Optimal preventive maintenance of a production system with an intermediate buffer," European Journal of Operational Research, vol. 168, no. 1, pp. 86-99, 2006.

[13] F. C. Gómez de León Hijes and J. J. R. Cartagena, "Maintenance strategy based on a multicriterion classification of equipments," Reliability Engineering and System Safety, vol. 91, no. 4, pp. 444451, 2006.

[14] M. Carnero, "An evaluation system of the setting up of predictive maintenance programmes," Reliability Engineering and System Safety, vol. 91, no. 8, pp. 945-963, 2006.

[15] M. Savsar, "Reliability analysis of a flexible manufacturing cell," Reliability Engineering and System Safety, vol. 67, no. 2, pp. 147$152,2000$.

[16] M. Savsar and M. Aldaihani, "Modeling of machine failures in a flexible manufacturing cell with two machines served by a robot," Reliability Engineering and System Safety, vol. 93, no. 10, pp. 1551-1562, 2008.

[17] M. Savsar, "Performance analysis of an FMS operating under different failure rates and maintenance policies," International Journal of Flexible Manufacturing Systems, vol. 16, no. 3, pp. 229249, 2005.

[18] M. Savsar, "Effects of maintenance policies on the productivity of flexible manufacturing cells," Omega, vol. 34, no. 3, pp. 274$282,2006$.

[19] M. Savsar, "Buffer allocation in serial production lines with preventive and corrective maintenance operations," Kuwait Journal of Science and Engineering, vol. 33, no. 2, pp. 253-266, 2006. 

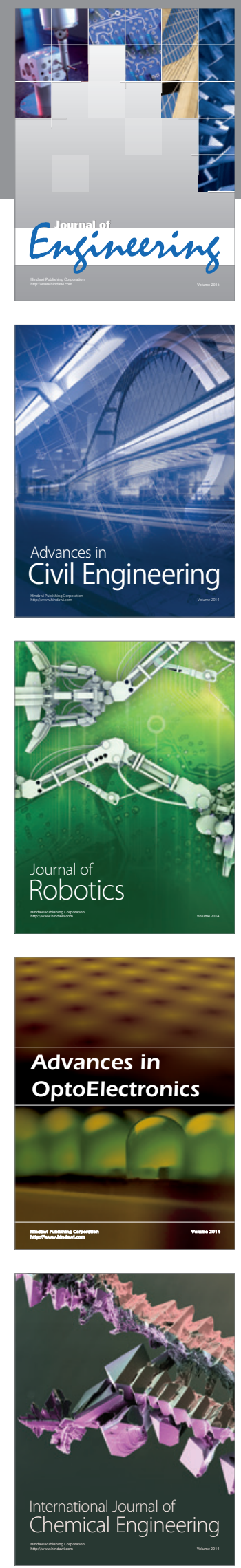

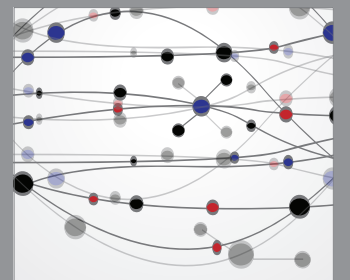

The Scientific World Journal
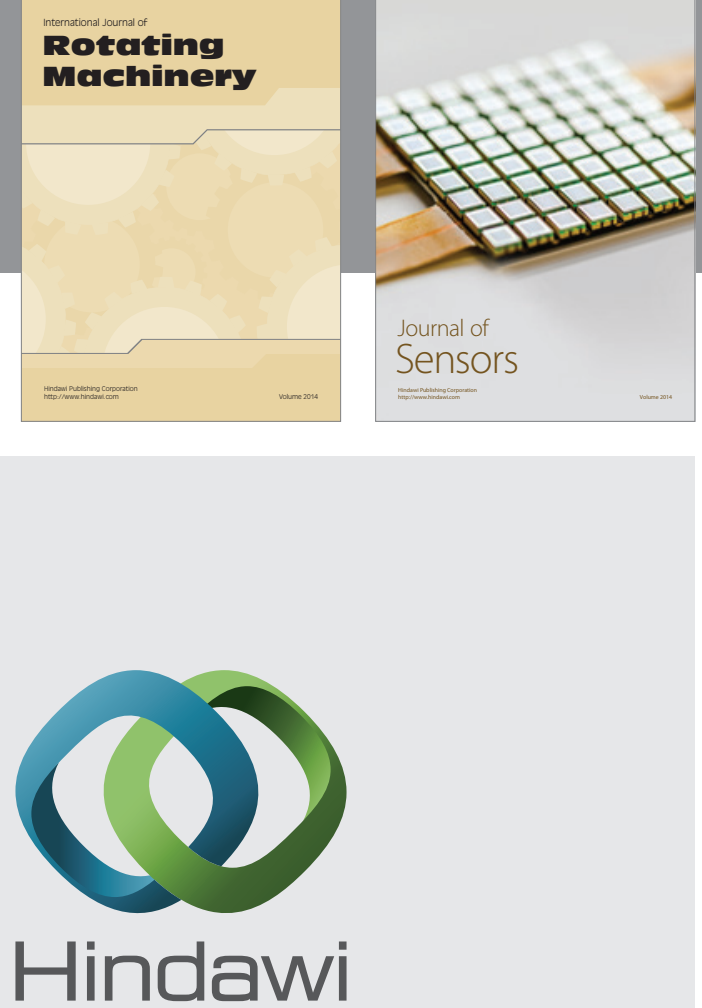

Submit your manuscripts at http://www.hindawi.com
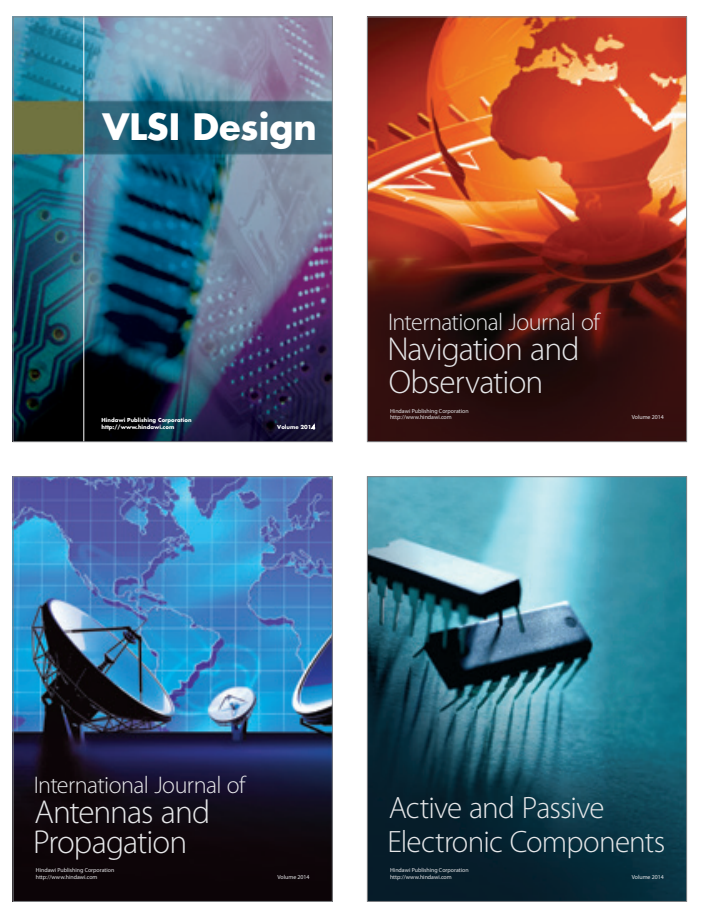
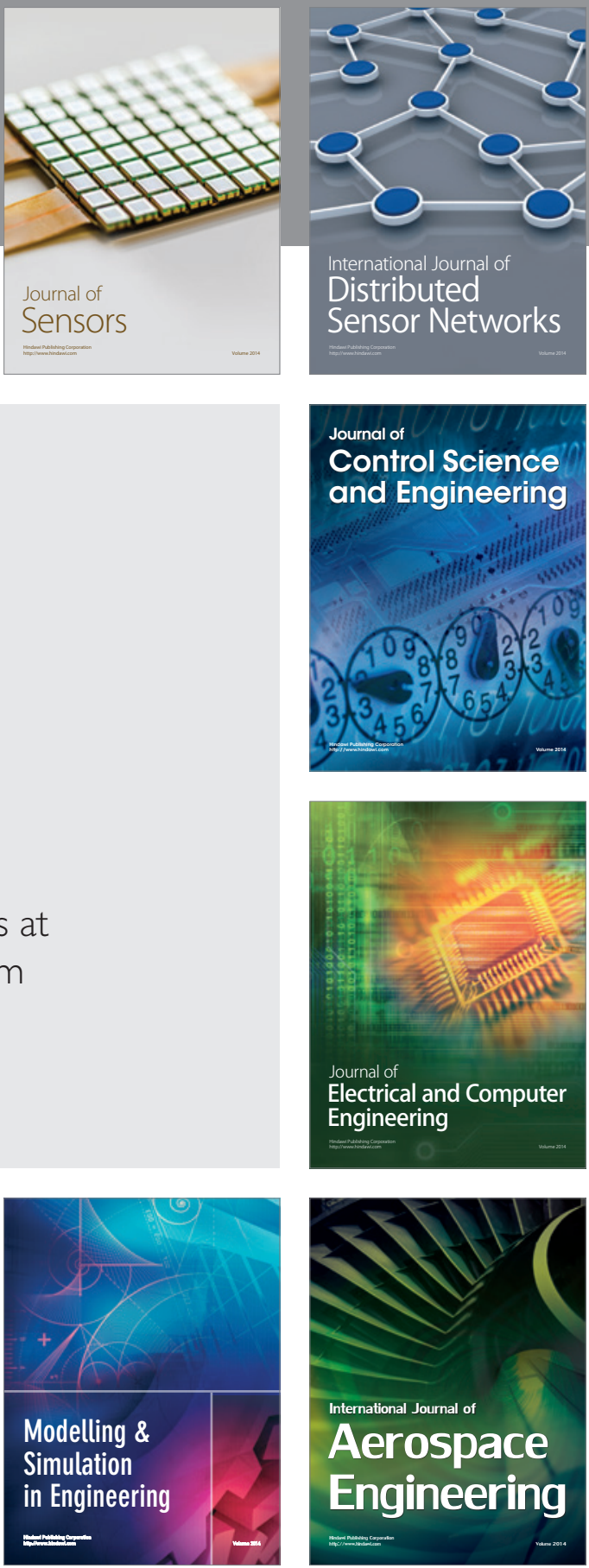

Journal of

Control Science

and Engineering
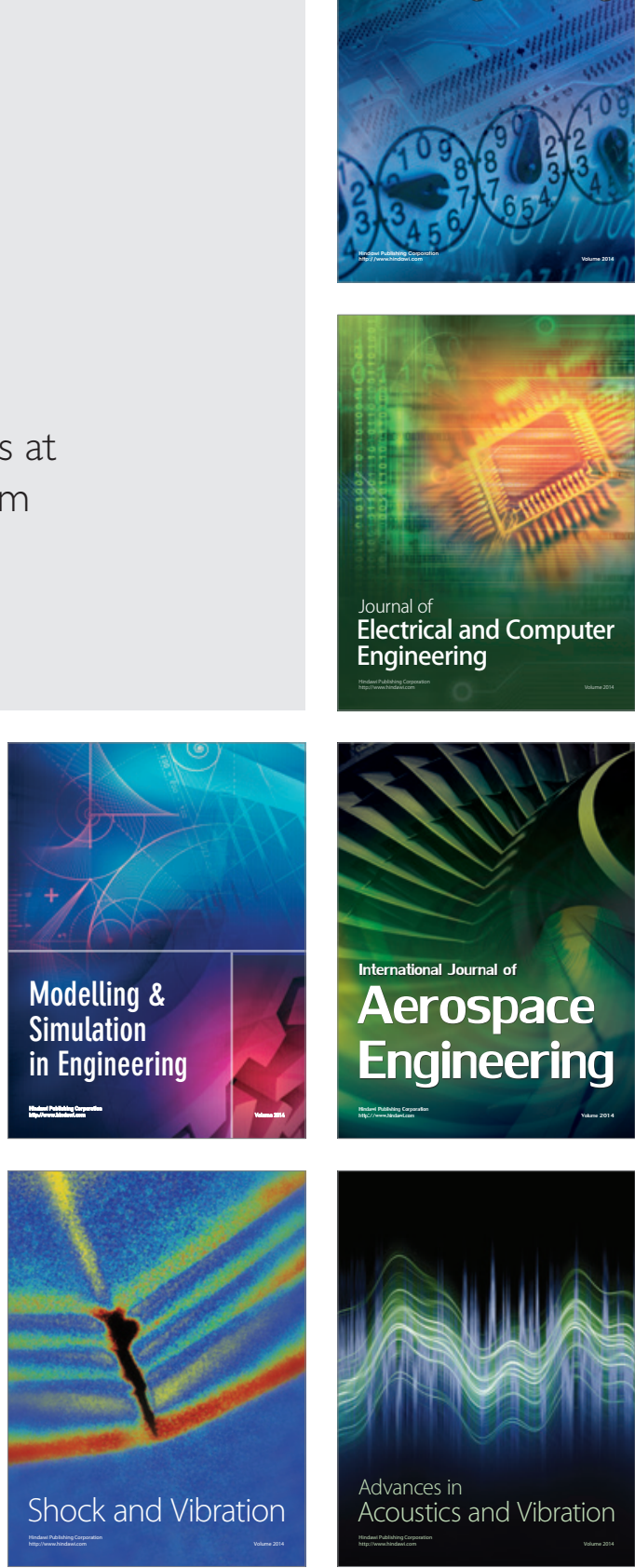